FTIR study of a chiral tilted SmA liquid crystalline phase

This article has been downloaded from IOPscience. Please scroll down to see the full text article. 2002 Europhys. Lett. 57184

(http://iopscience.iop.org/0295-5075/57/2/184)

View the table of contents for this issue, or go to the journal homepage for more

Download details:

IP Address: 134.226.1.229

The article was downloaded on 01/07/2010 at 12:00

Please note that terms and conditions apply. 
Europhys. Lett., 57 (2), pp. 184-190 (2002)

\title{
FTIR study of a chiral tilted SmA liquid crystalline phase
}

\author{
O. E. Kalinovskaya ${ }^{1}$, Yu. P. Panarin ${ }^{2}$ and J. K. ViJ ${ }^{1}\left(^{*}\right)$ \\ 1 Advanced Materials Laboratory, Department of Electronic and Electrical Engineering \\ Trinity College, University of Dublin - Dublin 2, Ireland \\ 2 School of Electronic $\&$ Communications Engineering, Dublin Institute of Technology \\ Kevin street, Dublin 8, Ireland
}

(received 26 March 2001; accepted in final form 26 October 2001)

PACS. 42.70.Df - Liquid crystals.

PACS. 61.30.Gd - Orientational order of liquid crystals; electric and magnetic field effects on order.

PACS. 78.30.-j - Infrared and Raman spectra.

\begin{abstract}
In this paper we present the results of the polarized infrared spectroscopy as evidence for the existence of a chiral analogue of the SmA phase of the de Vries type. In this phase, the molecular director in the smectic layers is tilted with respect to the smectic layer normal. The dichroic ratio is found to increase when the voltage crosses a threshold, and reaches a saturation value where all the directors of different layers are interpreted to be aligned perpendicular to the direction of the electric field. The possible molecular structure of de Vries $\mathrm{SmA}^{*}$ phase is discussed.
\end{abstract}

Introduction. - It is well known that the smectic layer spacing in all of the tilted smectic phases is lower than in the orthogonal smectic phases due to the molecular tilt. Nevertheless, in 1972, Diele et al. [1] reported a SmA phase, with the same layer spacing as a non-chiral SmC. Later on, in several publications the unusual SmA-SmC phase transition without smectic layer shrinkage was observed both in the non-chiral [2-5] and recently in chiral smectic LCs [6-9]. The non-chiral analogue of this phase was suggested and investigated first by de Vries in 1974 [2] (called $\mathrm{A}_{3}$ ). The smectic $\mathrm{C}_{3}$ phase on heating would yield $\mathrm{A}_{3}$, and this change-over at the $\mathrm{C}_{3}-\mathrm{A}_{3}$ transition temperature occurs without an alteration in the smectic layer spacing and therefore without a change in the molecular tilt angle. It was suggested that "in this phase the tilted layers are stacked in a random fashion" [2]. In other words, in the smectic $\mathrm{A}_{3}$ phase, the molecules are tilted with respect to the smectic layer normal, similar to those in the $\mathrm{SmC}^{*}$ phase, but the tilt directions in different smectic layers are randomly oriented, or uncoupling of the tilt directions from layer to layer occurs. The theoretical explanation of this de Vries phase has been developed in terms of a diffuse-cone model [5]. On the other hand, the same property (unshrinkable layer spacing) can be explained by a structure, where the molecular tilt direction is not correlated even in each single smectic layer. Due to the absence of chirality, and consequently the absence of local spontaneous polarization, both possible structures cannot be

$\left(^{*}\right)$ E-mail: jvij@tcd.ie

(c) EDP Sciences 
distinguished from each other and from the orthogonal SmA phase. In chiral smectic liquid crystals these phases exhibit a pronounced electroclinic effect [8]. Moreover, other smectic materials with a large electroclinic coefficient $[10,11]$ may exhibit the same phenomenon. Two properties, the unshrinkable smectic layers at the $\mathrm{SmA}_{-} \mathrm{SmC}^{*}$ transition and a large electroclinic coefficient, appear to be the essential features of these materials, though differing explanations have been given. The possible structure of the tilted SmA phase is similar to a new class of phases [12] that is predicted to occur in weakly coupled, three-dimensional stacks of two-dimensional $X Y$ systems or sliding phase. This is also supported by recent computer simulations of smectic ordering in liquid crystals [13] composed of bent-rod strongly angled molecules interacting through only a soft repulsive potential, arising from the steric interactions. The interlayer coupling and the interdigitation of molecules in adjacent layers is neglected. This reveals a $\mathrm{SmC}$ phase with direction of molecular tilt varying from layer to layer. The existence of a "sliding phase" is also predicted by the axial next-nearest-neighbor interactions (ANNNI) model [14], where the competitive disordering effect of the thermal fluctuations is greater than the correlation between neighboring smectic layers.

In this paper we present the results of the polarized infrared measurements of the sample under investigation. The results of the effect of the applied voltage on the absorbance profiles are shown for $\mathrm{SmC}^{*}$ and de Vries $\mathrm{SmA}^{*}$ phases. We determine the dichroic ratio as a function of the applied voltage. The infrared results on the molecular tilt angle are compared with those found using electro-optic measurements in our earlier paper [9].

Experimental. - The sample under investigation is MC513. This is the second compound of a three-component Tokyo mixture investigated in ref. [15]. The molecular structure of this compound is

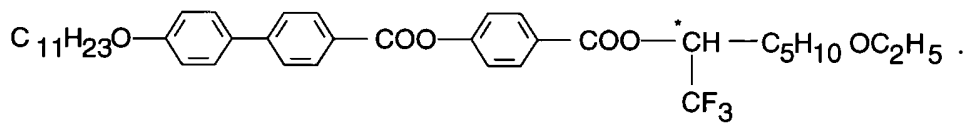

From the phase diagram, presented in ref. [15], the phase sequence of this compound was as follows:

$$
\mathrm{Cr}-35{ }^{\circ} \mathrm{C}-\mathrm{SmC}_{A}{ }^{*}-73{ }^{\circ} \mathrm{C}-\mathrm{SmC}^{*}-81.7{ }^{\circ} \mathrm{C}-\mathrm{SmA}-84.5^{\circ} \mathrm{C}-\mathrm{Is} \text {. }
$$

The sample cell for the infrared measurements consisted of two $\mathrm{CaF}_{2}$ windows coated with conducting layers of indium tin oxide (ITO). We made investigations on a homogeneously aligned $5 \mu \mathrm{m}$ cell. The orientant RN1266 (Nissan Chemicals Ind. Ltd.) was used as an alignment agent. The cell was filled with the liquid crystal in its isotropic phase by the capillary effect and the sample was trained to produce a uniform structure in order to attain a high dichroic ratio. A Bio-Rad spectrometer FTS-60A with $2 \mathrm{~cm}^{-1}$ resolution and averaging over 32 scans was used to record the spectra. Infrared spectra were measured as a function of the polarizer rotation angle under the application of a bias voltage at temperatures of $81.2^{\circ} \mathrm{C}$ and $83.5^{\circ} \mathrm{C}$. These temperatures correspond to $\mathrm{SmC}^{*}$ and $\mathrm{SmA}$ phases, respectively. However, the latter phase $(\mathrm{SmA})$ is being interpreted as a non-orthogonal de Vries $\mathrm{SmA}^{*}$ phase.

Results and discussion. - In our investigation we focus our attention only on the phenyl ring C-C stretching vibrations centred at about $1600 \mathrm{~cm}^{-1}$ mainly because its transition dipole moment is almost parallel to the long molecular axis. This is used to find out the structural arrangement of the molecules on a microscopic level.

The infrared properties of the $\mathrm{SmC}^{*}$ phase are found to correspond to those of a conventional $\mathrm{SmC}^{*}$ phase. Figure 1 presents the absorbance profile $A(\Omega)$ for the phenyl ring C-C 


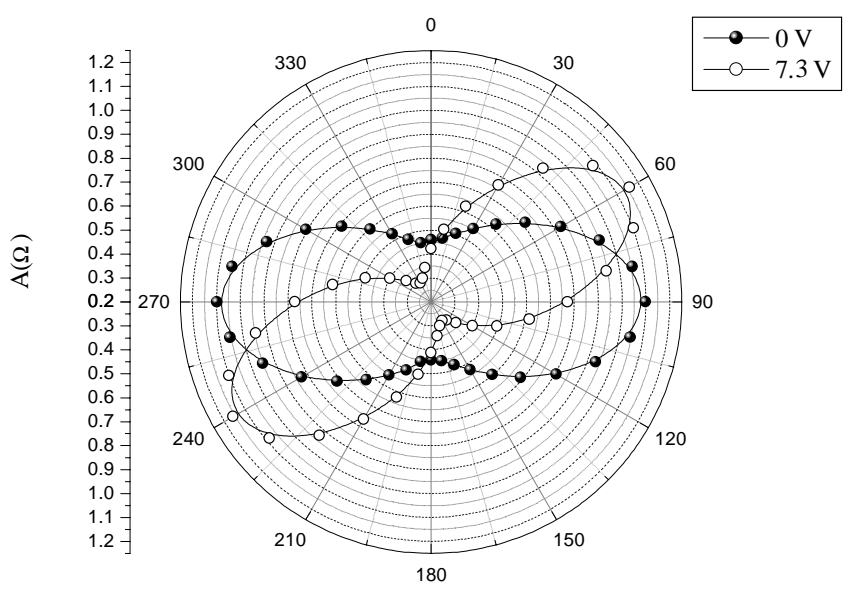

Fig. 1 - Absorbance profiles of phenyl ring $\mathrm{C}-\mathrm{C}$ stretching vibrations in the $\mathrm{SmC}^{*}$ phase with and without electric field at a temperature of $81.2^{\circ} \mathrm{C}$ (the electric field is perpendicular to the page plane).

stretching vibration as a function of the polarizer rotation angle for zero and saturated voltage in the $\mathrm{SmC}^{*}$ phase. The application of the bias voltage unwinds the ferroelectric helix resulting in a rotation of the absorbance profile by an angle " $+\Omega_{0}$ " or " $-\Omega_{0}$ " depending on the polarity of the external field. When the helix is unwound $\Omega_{0}$ reaches $\Omega_{\max }$. The absorbance $v s$. polarizer rotation angle for different values of applied voltage was fitted to the following equation $[16,17]$ :

$$
A(\Omega)=-\log _{10}\left[10^{-A_{\|}}+\left(10^{-A_{\perp}}-10^{-A_{\|}}\right) \sin ^{2}\left(\Omega-\Omega_{0}\right)\right],
$$

where $A_{\|}$and $A_{\perp}$ are the maximum and minimum values of absorbance, $\Omega$ is the polarizer angle, $\Omega_{0}$ is the angle of maximal absorbance. The angle $\Omega_{0}$ obtained from the fitting of infrared spectra is found to be equal to the optical tilt angle [9]. Figure 2 shows the dichroic ratio $\left(=A_{\|} / A_{\perp}\right)$ and the optical tilt angle $\Omega_{0} v s$. the applied voltage.

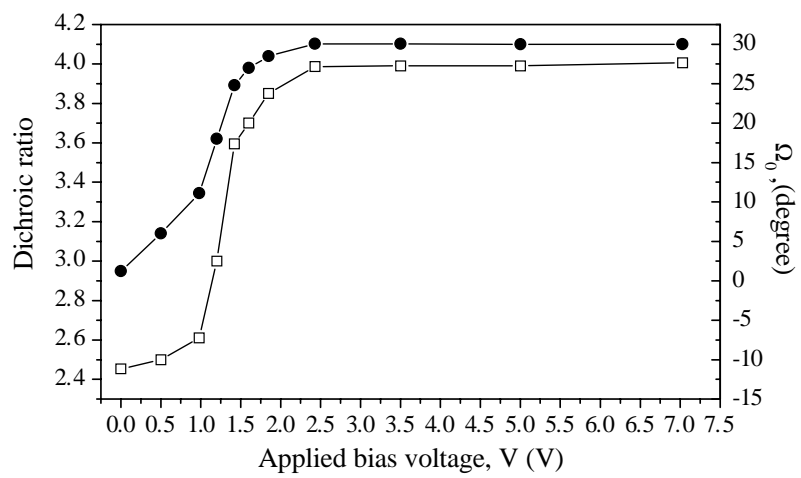

Fig. 2 - Dichroic ratio (filled circles) and angular shift (open squares) as a function of applied voltage for a phenyl ring $\mathrm{C}-\mathrm{C}$ stretching vibration in the $\mathrm{SmC}^{*}$ phase. The temperature is the same as in fig. 1. 


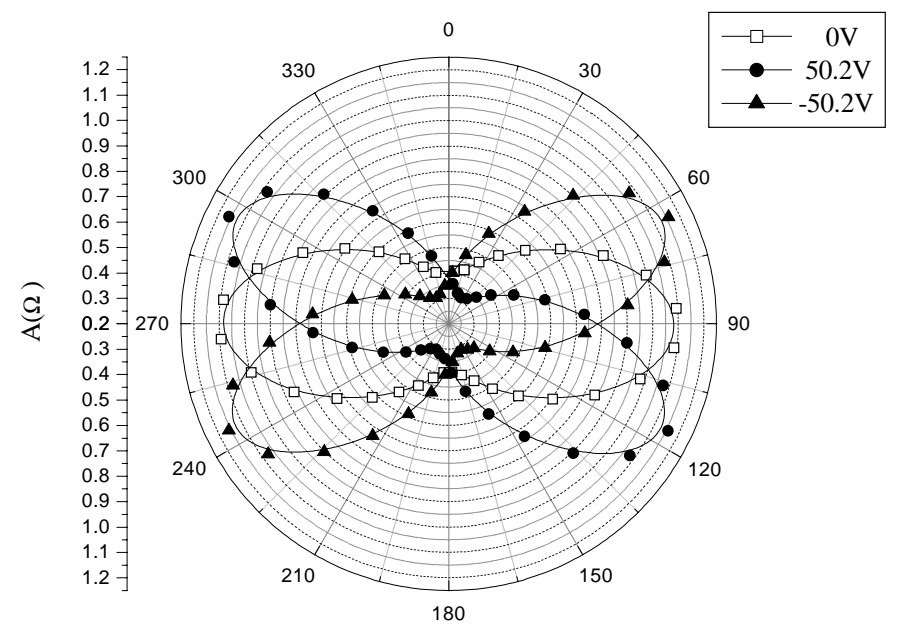

Fig. 3 - Absorbance profiles of phenyl ring C-C stretching vibrations in the de Vries SmA* phase with and without electric field at a temperature of $83.5^{\circ} \mathrm{C}$ (the electric field is perpendicular to the page plane).

A distortion in the helical structure with voltage causes an increase in the dichroic ratio until it reaches the maximum value corresponding to a completely unwound ferroelectric phase. The maximum dichroic ratio was found to be equal to 3.98 and $\Omega_{\max }$ is $\sim 29^{\circ}$; the latter is in good agreement with the result of the optical tilt angle measurements [9]. $\Omega_{\max }$ is the saturation value of $\Omega_{0}$.

Similar measurements in the SmA phase of this material show extremely intriguing results. The most unusual is the behavior of the dichroic ratio, which is found to be dependent on the applied electric field. Figure 3 presents a polar plot for the high-temperature phase in the absence of an electric field and under positive and negative voltages where the absorbance reaches a saturation value. The saturated voltage is found to be approximately $50-55 \mathrm{~V}$ for a temperature of $83.5^{\circ} \mathrm{C}$. The fitted parameters of the absorbance profiles, i.e., the dichroic ratio and the angular shift, are plotted in fig. 4.

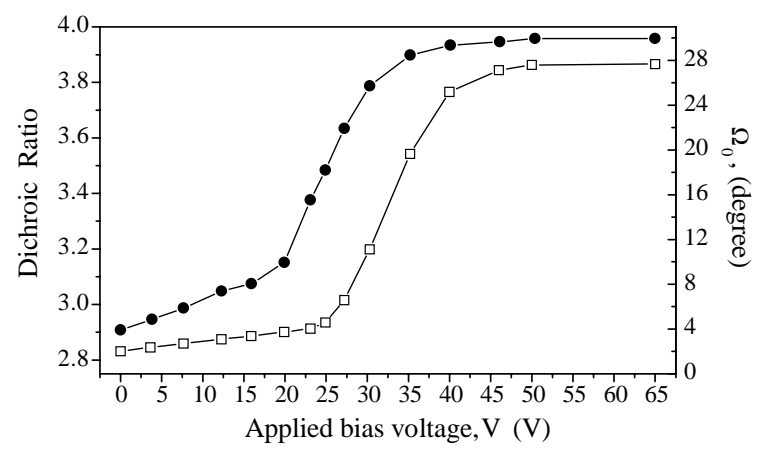

Fig. 4 - Dichroic ratio (filled circles) and angular shift (open squares) as a function of applied voltage for phenyl ring C-C stretching vibrations in the de Vries SmA* phase. The temperature is the same as in fig. 3 . 
The results for the angular shift determined from the IR agree with those from the optical measurements of the apparent tilt angle [9]. The changes in $\Omega_{0}$ at voltages below the threshold are similar to the electroclinic effect in the SmA phase, where the electrically induced angular shift (apparent/switching) tilt angle increases almost linearly with the electric field. However, at some threshold voltage, $\Omega_{0}$ jumps to a saturated value $\left(\approx 27^{\circ}\right)$. This result is in contradiction to that for a conventional SmA phase. In the optical tilt angle measurements [9] we find that the threshold voltage increases rapidly with the increase in temperature, while the saturation angle remains almost independent of temperature. This is typical for a smectic liquid crystal with a direct $\mathrm{SmC}^{*}$-Is phase transition, where the smectic tilt angle is almost independent of temperature. This observation indicates that the saturation value of the switching angle corresponds to the thermodynamic molecular tilt angle in the smectic layers. The threshold voltage corresponding to an abrupt increase in the dichroic ratio is interpreted as the voltage where all the molecules start getting aligned perpendicular to the direction of the field.

The similar shape of the field-induced tilt angle has been interpreted by the field-induced SmA-SmC* phase transition or by a large high-order coefficient $\left(\Theta^{6}\right)$ in the Landau free-energy expansion [7]. Nevertheless, these two interpretations contradict the voltage dependence of the dichroic ratio (fig. 4). If it was a normal $\mathrm{SmA}_{-} \mathrm{SmC}^{*}$ field-induced transition, then the dichroic ratio is not likely to be greater than that for a SmA phase, and furthermore such a significant increase in the dichroic ratio is not expected. In the case of a large high-order coefficient $\left(\Theta^{6}\right)$ in the Landau free energy, the shape of the curve will be similar with the exception that a saturation value in the tilt angle, as in fig. 4 , will not be reached. The tilt angle will then continue to increase with a lower slope for voltages greater than the threshold.

The fact that the dichroic ratio first slowly (almost linearly) increases with the electric field and then suddenly rises and reaches a saturation value suggests that the phase under discussion is a de Vries tilted smectic phase.

A simple simulation can also support this. In the absence of electric field the dichroic ratio of a de Vries phase must be lower than that in the unwound state and it can be estimated by taking into account the absorbance profile for the unwound $\mathrm{SmC}^{*}$ phase and integrating it for different values of $\varphi$ to introduce an arbitrary distribution of molecular tilt direction, as in the following equation:

$$
A(\Omega)=\frac{1}{2 \pi} \int_{0}^{2 \pi}-\log _{10}\left[10^{-A_{\|}}+\left(10^{-A_{\perp}}-10^{-A_{\|}}\right) \sin ^{2}\left[\left[\Omega-\Omega_{0} \cdot \cos (\varphi)\right] \frac{\pi}{180}\right]\right] \mathrm{d} \varphi .
$$

This equation can be applied to both the helical $\mathrm{SmC}^{*}$ phase and the de Vries $\mathrm{SmA}$ * phase because the aperture of the infrared beam is much greater that the helical pitch. Taking the following experimental parameters for the unwound random phase: $A_{\|}=1.144, A_{\perp}=0.296$, $A_{\|} / A_{\perp}=3.86, \Omega_{\max }=27^{\circ}$ in eq. (2), the dichroic ratio for the undisturbed random structure comes out to be 2.8. This is in excellent agreement with the experiment. Nevertheless, a similar approximation for the helical $\mathrm{SmC}^{*}$ phase (again on taking the experimental results of $A_{\|}=0.96, A_{\perp}=0.241, A_{\|} / A_{\perp}=3.98, \Omega_{\max }=29^{\circ}$ ) gives a dichroic ratio of 2.7 which is higher than the experimental value of 2.5. This discrepancy can be easily explained by the well-known fact that a helix in a planar cell is distorted by the surface. In other words, some of the molecules near the molecular-surface interface lie in the surface plane. This causes a decrease in the experimental value compared to the theoretical one. On the other hand, the random structure, appearing to be stronger than the helical $\mathrm{SmC}^{*}$, is almost undistorted. In this case the experimental and theoretical values are of the same magnitude. 
Finally, there are two possible structures suggested for the de Vries phase. One of these (say Type I) is a phase where the molecules are randomly tilted in the same smectic layer and there is only short-range order in the tilting sense. The second possible structure (Type II) is similar to a sliding phase [12] where the molecules in a smectic layer are tilted in the same direction, but the tilting directions (C-directors) from layer to layer are randomly distributed. In this case the local symmetry of the smectic layer $\left(\mathrm{C}_{2}\right)$ is the same as for SmC* and the smectic layer possesses local spontaneous polarization. Both structures satisfy the experimental results and therefore they cannot be distinguished from each other by FTIR analysis. Nevertheless, we believe the sample is more likely to be a de Vries (Type II) phase; this conclusion follows from the shape of the field-induced dependence of the apparent tilt angle [9] and dichroic ratio.

Several different approaches (Monte Carlo simulations [13] and mean-field theory [18]) showed that the Type-I de Vries phase possesses a smooth, Langevin-type field-induced dependence of the apparent tilt angle, without inflection point, so that $\mathrm{d}^{2} \psi / \mathrm{d} E^{2}<0$, where $\psi$ is the apparent tilt angle. Recently, such a smooth dependence has been obtained experimentally in other tilted SmA (de Vries) samples [8]. The shape of the field-induced dependence of the dichroic ratio and the apparent tilt angle [9] of MC513 reveal an abrupt increase. At this point microscopic observation detects the coexistence of two types of domains: one of these is a distorted de Vries structure and the other is an unwound $\mathrm{SmC}$ with a saturated tilt angle. The shape of the domains is strongly elongated in the direction of the smectic layer plane. Such a scenario is more appropriate for the de Vries Type-II structure (sliding phase).

In such a structure the local spontaneous polarization vectors in different smectic layers are randomly distributed while these are still coupled [12]. The application of an external electric field causes a deformation in the random distribution of the local polarization of smectic layers and induces some order (macroscopic polarization, apparent tilt). At some threshold value of the applied voltage the net spontaneous polarization of some smectic layers is oriented in the direction of the electric field. This will enable the neighboring smectic layers to turn in the same direction and therefore form specific elongated domains. This situation is similar to the appearance of "dechiralization" lines (or domains) in the conventional helical SmC* cells.

Finally we conclude that the investigation using Infrared Spectroscopy supports the existence of a de Vries SmA phase and the experimental results for the MC513 are more appropriate possibly for a Type-II de Vries phase. Additional investigations employing different experimental techniques should clarify the nature of this phase.

We thank Prof. A. FukUda for giving us this material and for fruitful discussions. Prof. N. A. Clark is also thanked for useful discussions. PRF of the ACS is acknowledged for the research grant PRF No. 33221-HC7. The Trinity College and DIT are thanked for a co-operative SEED grant.

\section{REFERENCES}

[1] Diele S., Brand P. and Sackmann H., Mol. Cryst. Liq. Cryst., 16 (1972) 105.

[2] De VRies A., Abstracts of the 5th International Liquid Crystals Conference, Stockholm (1974) p. 150.

[3] De Vries A., Mol. Cryst. Liq. Cryst., 41 (1977) 27.

[4] De Vries A., Ekachai A. and Spielberg N., Mol. Cryst. Liq. Cryst. Lett., 49 (1979) 143.

[5] De Vries A., J. Chem. Phys., 71 (1979) 25. 
[6] Radcliffe M. D., Brostrom M. L., Epstein K. A., Rappaport A. G., Thomas B. N., Shao R. and Clark N. A., Liq. Cryst., 26 (1999) 789.

[7] Giesselmann F., Zugenmaier P., Dierking I., Lagerwall S. T., Stebler B., Kaspar M., Hamplova V. and Glogarova M., Phys. Rev. E, 60 (1999) 598.

[8] Spector M. S., Heiney P. A., Naciri J., Weslowski B. T., Holt D. B. and Shashidhar R., Phys. Rev. E, 61 (2000) 1579.

[9] Panarin Yu. P., Panov V., Kalinovskaya O. E. and Vij J. K., J. Mater. Chem., 9 (1999) 2967.

[10] Bahr Ch. and Heppke G., Phys. Rev. A, 39 (1989) 5459.

[11] Bahr Ch. and Heppke G., Ferroelectrics, 114 (1991) 311.

[12] O'Hern C. S., Lubensky T. C. and Toner J., Phys. Rev. Lett., 83 (1999) 2745.

[13] Xu J., Selinger R. L. B., Selinger J. V., Ratna B. R. and Sashidhar R., Phys. Rev. E, 60 (1999) 5584.

[14] Yamashita M. and Miyazima S., Ferroelectrics, 148 (1993) 1.

[15] Inui S., Immura N., Suzuki T., Iwane H., Miyachi K., Takanishi Y. and Fukuda A., J. Mater. Chem., 6 (1996) 671.

[16] Jang W. G., Park C. S., Maclennan J. E., Kim K. H. and Clark N. A., Ferroelectrics, 180 (1996) 213.

[17] Kocot A., Wrzalik R. and Vij J. K., Liq. Cryst., 21 (1996) 147.

[18] Clark N. A., Bellini T., Shao R.-F., Coleman D., Bardon S., Link D. R., Maclennan J. E., Chen X.-H., Wand M. D., Walba D. M., Rudquist P. and Lagerwall S.T., to be published in Phys. Rev. Lett. (2001). 